\title{
A Case Report with Literature Review: Parasitic Leiomyoma
}

\author{
Salem Al Zoghbi, K. Keriakos, Saad El Daou, J. Darido* \\ Saint Georges University Hospital, Achrafieh, Beirut, Lebanon \\ Email: ${ }^{\star}$ jesydarido@hotmail.com
}

How to cite this paper: Al Zoghbi, S., Keriakos, K., El Daou, S. and Darido, J. (2019) A Case Report with Literature Review: Parasitic Leiomyoma. Journal of Cancer Therapy, 10, 28-35. https://doi.org/10.4236/jct.2018.101003

Received: November 5, 2018

Accepted: January 8, 2019

Published: January 11, 2019

Copyright $\odot 2019$ by author(s) and Scientific Research Publishing Inc. This work is licensed under the Creative Commons Attribution International License (CC BY 4.0).

http://creativecommons.org/licenses/by/4.0/ (c) (i) Open Access

\begin{abstract}
Leiomyoma is a benign tumour composed mainly of smooth muscle cells. It contains varying amount of fibrous connective tissues. Parasitic leiomyoma is a rare manifestation of uterine leiomyoma defined as a pedunculated subserosal fibroid that undergoes torsion and detaches from the uterus. We are reporting a case of parasitic leiomyoma in a $55 \mathrm{Y} / \mathrm{O}$ female patient that had already given her consent for the case to be published. She presented with increased abdominal girth since 6 months and body weight and consulting for possible bariatric surgery 1 month prior to presentation. Ultrasonography revealed a large heterogeneous mass starting from the pelvic region to the middle and upper abdomen of more than $37 \mathrm{~cm}$ compatible with an adnexal mass. On abdominopelvic MRI: presence of an enlarged heterogeneous mass that seems taking its origin from the right ovary, an exploratory laparotomy was performed. Identification and complete excision of a huge mass of cystic and solid component adherent to the uterus and the mesentery of the small bowel. Histopathologically mass was confirmed as a hyelanized leiomyoma with a focal necrobiosis with no signs of malignity. Parasitic leiomyomas are rare tumors. Imaging modalities help in diagnosis but not always. The definitive treatment is surgical excision, presenting this case due to its rarity and diagnostic difficulties posed.
\end{abstract}

\section{Keywords}

Parasitic, Leiomyoma, Rare, Tumors, Uterus

\section{Introduction}

Parasitic leiomyoma is a rare manifestation of uterine leiomyoma. It is also known as wandering fibroid, Migrating fibroid, Ectopic fibroid [1]. It's defined as a pedunculated subserosal fibroid that undergoes torsion and detaches from 
the uterus.

It was first described by Kelly and Cullen in 1909: "a myoma that has for some reason become partially or almost completely detached from the uterus and received their main blood supply from another source" [2].

We are reporting a case of parasitic leiomyoma in a $55 \mathrm{Y} / \mathrm{O}$ female patient that had already given her consent for the case to be published.

\section{Case Presentation}

A 55 y/o female patient presenting with increased abdominal girth since 6 months and body weight. She consulted her endocrinologist about her gain weight for possible bariatric surgery 1 month prior to presentation. An abdominopelvic ultrasound was ordered and showed the presence of a large heterogeneous mass starting from the pelvic region to the middle and upper abdomen of more than $37 \mathrm{~cm}$. It contains large cystic structures, highly suspicious, hypervascularized and compatible with an adnexal mass requiring scanner imaging, surgical excision and pathology examination. Her past medical history includes hypertension and in her past surgical history, a bilateral inguinal hernia repair. She's a non smoker. Upon presentation her vitals were stable (Temperature: $36^{\circ} \mathrm{C}$; Heart Rate: 62/minutes; Blood Pressure: 130/80). On physical exam, we noted the presence of a large abdomen with a large palpable mass in the mid pelvis reaching the upper abdomen. Lab tests done: WBC: 6700 (neutrophils: 53\%); Hb: 14.1; PT, PTT Normal; Creatinine Normal; LFT's Normal; CRP 5; Tumor markers ( CEA CA125 CA19-9 AFP BCG) were not financially covered. Abdomino pelvic CT scannoted the presence of an enlarged heterogeneous mass measuring $31 \mathrm{~cm}$ (sagittal cut) and $36 \mathrm{~cm}$ (axial cut) suppressing digestive structures, in contact with some ileal loops with an impact over the bladder dome and the uterine fundus and a fatty border without signs of invasion. There is also a small effusion in the douglas; no adenopathy is seen; no peritoneal carcinomatosis; ovaries are not seen. On abdominopelvic MRI: presence of an enlarged heterogeneous mass that seems taking its origin from the right ovary. Presence of a noduleis at the right posterolateral side wall, the uterus and left ovary are normal. A decision was taken for exploratory laparotomy and possible debulking surgery. Urology consulted for JJ insertion. Hereby the operative report:

- Midline incision from xyphoid to suprapubic;

- Identification of a huge mass of cystic and solid component adherent to the uterus and the mesentery of the small bowel (Figure 1, Figure 2);

- Ovaries were free;

- Identification of fluid in the douglas, aspirated and sent for cytology studies;

- Complete excision of the mass by liberating it from the mesentery and the uterus using ligasure (Figure 3, Figure 4);

- Specimen sent to frozen section (Figure 5);

- Identification of a small nodular lesion (infracentimetric) in the liver; 
- Excision of the nodule and specimen sent to pathology;

- Frozen section returned inconclusive with atypical fusiform cells most probably originating from the uterus (leiomyosarcoma?);

- Total abdominal hysterectomy (including the cervix) with bilateral oophorectomy and salpingectomy done (Figure 6);

- Hemostasis reassured;

- Insertion of a large hemovac drain in douglas;

- Closure of the Abdomen;

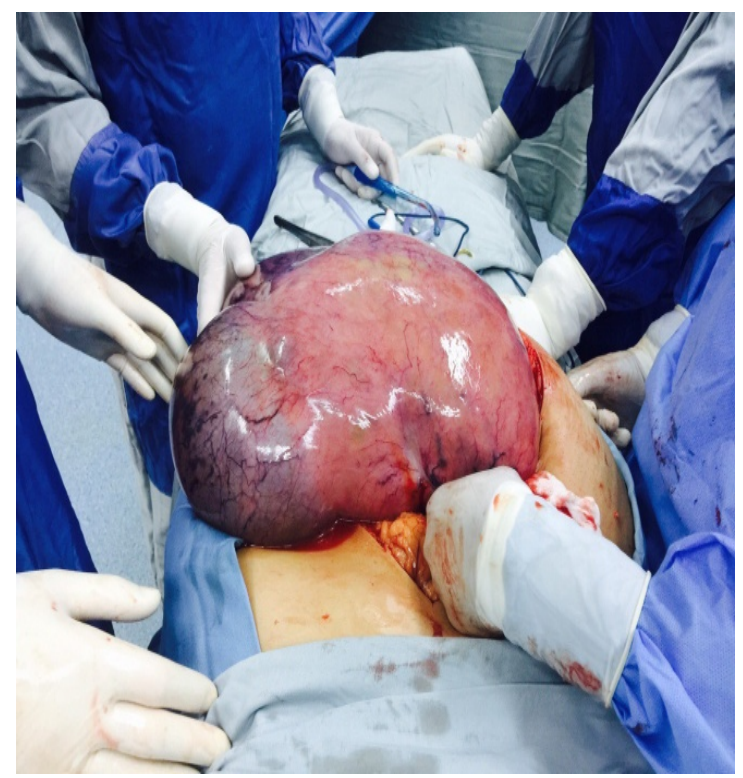

Figure 1. Identification of a huge mass adherent to the uterus and the mesentery of the small bowel.

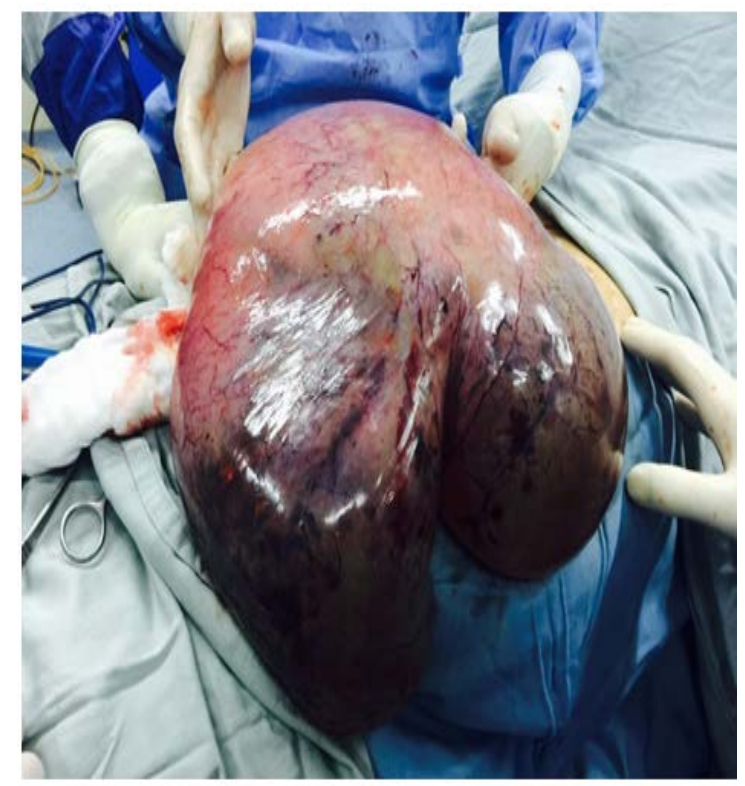

Figure 2. The mass is formed of cystic and solid components. 


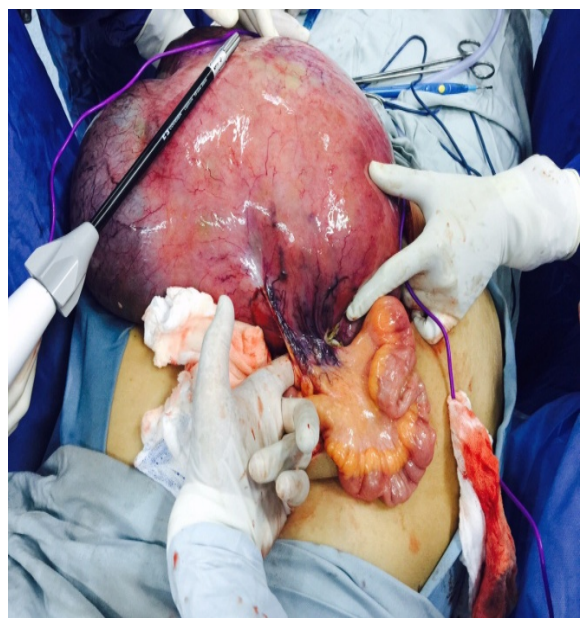

Figure 3. Dissection started for liberating the mass using ligasure.

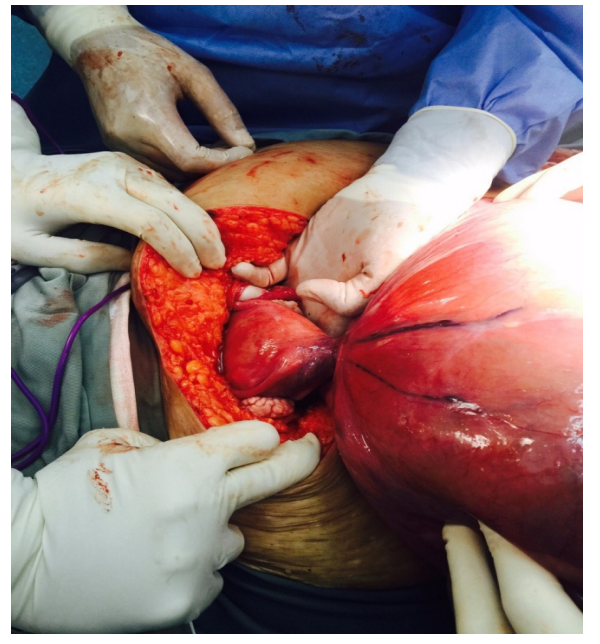

Figure 4. Complete excision of the mass by liberating it from the mesentery and the uterus.

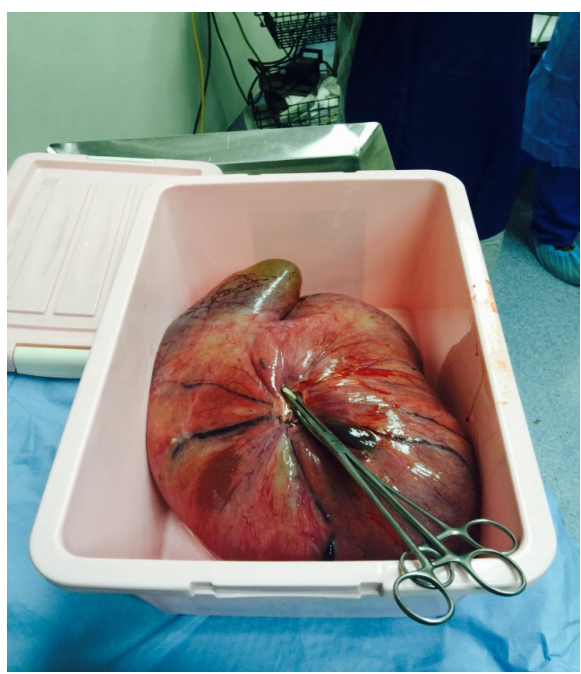

Figure 5. Specimen sent to frozen section. 


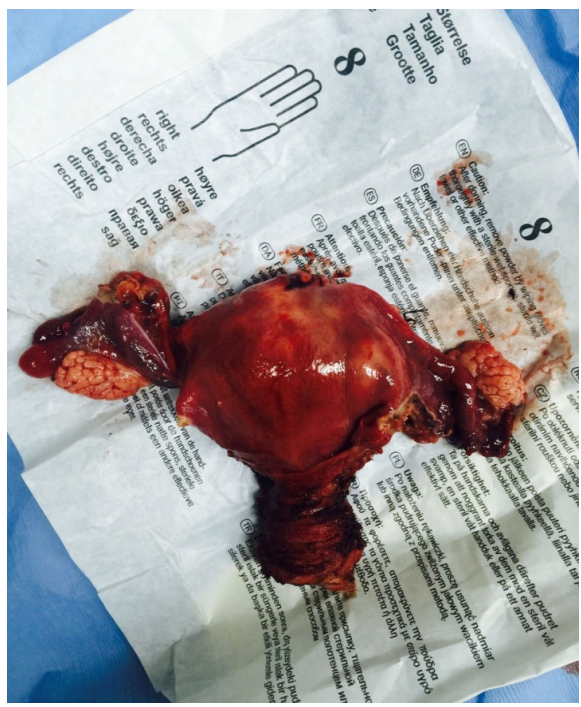

Figure 6. Total abdominal hysterectomy with bilateral oophorectomy and salpingectomy.

As for the follow-up of this patient, the postoperative course was uneventful. Patient was discharged home on day 5 post operatively. Pathology results that were out revealed the following: The pelvic mass is a hyalinized Leiomyoma with a focal necrobiosis measuring $40 \mathrm{~cm}$ of large axis. Malignity signs are absent. The hepatic nodule is a biliary canal fibroadenomatosis with no signs of malignity. As for the uterus and adnexa, presence of adenomyosis, leiomyomas and achronic metaplastic cervicitislocally erosive. The adnexa have no particularity and no signs of malignity. The peritoneal fluid has a reactional aspect.

\section{Discussion}

leiomyoma is a benign tumour composed mainly of smooth muscle cells [3]. It contains varying amount of fibrous connective tissue. An incidence of $50 \%$ was found at post-mortem examinations [4]. The incidence increases with age 4.3 per 1000 woman-years for 25 to 29 years old and $22.5 \%$ for 40 to 44 year olds [5]. A higher incidence has been noted in African American than in Caucasian women. Leiomyomas are classified as: Submucosal, intramural, subserosal and transmural [6].

Parasitic leiomyoma sustains its growth through neovascularization from adjacent tissue (peritoneum, mesenterium, and omentum). As per FIGO classification system, parasitic fibroids have been categorized as Type 8 leiomyomas with no myometrial involvement and uterine attachment [5].

There are two mechanisms for development of parasitic leiomyoma, either iatrogenic implantation during surgery or extreme forms of subserosal leiomyoma torsion. The former may occur as a complication of laparoscopic surgery: Incomplete removal of leiomyoma fragments generated during surgery can result in regrowth in the abdominal cavity [7]. Several such cases have been reported after the use of electrical power morcellation. In contrast, parasitic leiomyoma 
resulting from subserosal leiomyoma torsion is a rare tumor of spontaneous onset: Subserosal leiomyoma twists around its pedicle and completely loses its blood supply; the peduncular connection is eventually interrupted, resulting in a free nodule that is re-fed from abdominal organs.

Peritoneal metaplasia is another theory that describes the pathogenesis of myomas in unexpected fields of abdomen. The development of multiple nodules on peritoneal surfaces is referred to as leiomyomatosis peritonealis disseminata which was first described in 1952 by Wilson et al. [8].

Parasitic leiomyoma was reported in the literature via 4 case reports:

Tejaswini Kale and associates [9] reported a case of a 45 years old female, Gravida 3, Para 3, Living 3 presenting with pain and heaviness in lower abdomen since 1year, increased per vaginal bleeding with passage of clots during menses associated with generalized weakness since 6 months. On physical examination, a distended lower abdomen with a $15 \times 10 \mathrm{~cm}$ mobile mass, firm in consistency, occupying the hypogastrium and the lower umbilical region more is at the right side. Based on history and clinical examination, the clinical diagnosis was set as a uterine fibroid with a separate pelvic mass, probably ovarian. Abdominopelvic ultrasound showed a $13 \times 09 \times 10 \mathrm{~cm}$ uterus size with multiple fibroids from which a large right sided pedunculated subserous fibroid of $12 \times 8$ $\mathrm{cm}$. Bilateral ovaries were normal. An exploratory laparotomy was done starting with a vertical midline infraumbilical incision and showing a big mass of $15 \times 10$ $\mathrm{cm}$ with firm in consistency, attached to peritoneum and omentum with NOattachment to uterus. Consequently, the diagnosis of parasitic fibroid was made on table. The mass was freed from omental and peritoneal attachment. Pedicle clamped, cut and ligated after confirming that there is no bowel adhesion or involvement. Note that a total abdominal hysterectomy was also done.

Mana Iida et al. [10] reported a 30-year-old woman presenting with severe abdominal pain and a low-grade fever. In her Past Medical History, a recurrent abdominal pain during the last pregnancy and pain continued for 1 month after delivery and spontaneously resolved without any medication. A transvaginal ultrasound was performed showing a homogeneous solid tumor $5 \mathrm{~cm}$ in diameter on the left anterior abdominal wall, completely separated from the uterus. On CT scan, the tumor surface was enhanced. On MRI, low signal intensity on both T1- and T2-weighted and both ovaries were clearly distinct from the tumor. On PET/CT scan, no significant glucose uptake was detected. The relationship between the omentum and the tumor was unable to be determined on imaging. Except for the tumor's complete segregation from the uterus, all findings were consistent with subserosal leiomyoma. On gastrointestinal and colon endoscopy, there are no abnormalities. On diagnostic laparoscopy, the tumor was tightly adhered to the omentum and the anterior left side of the peritoneum. An abundant vascularization was present from the omentum to the tumor surface. Both ovaries were normal in appearance and distinct from the tumor. The adhesions to the omentum and peritoneum were released. Note that the tumor was broadly 
hyalinized and partially calcified, which is consistent with degenerated uterine leiomyoma. It was therefore diagnosed as parasitic leiomyoma.

Ahmed S. Elagwany and associates [11] reported a 37-year-old woman, mother of two children presenting with bloating during feeding, abdominal pain and distention. She has normal and regular menstrual cycles with No associated vaginal discharge. Note that she was previously operated of a myomectomy six years ago. On physical examination: abdomen was distended with multiple masses that were felt all over the abdomen. An abdominopelvic ultrasound showed a bulky uterus with nearly $15 \times 15 \mathrm{~cm}$ large fundal subserousfibroid. Doppler blood flow study showed that the blood supply was away from the uterus. Ovaries, spleen, liver and pancreas were all normal. Exploratory laparotomy revealed: multi-lobulated fibroid like mass densely adherent to the greater omentum. It derived its blood supply from the greater omentum with a hugeserpentine and spider shaped blood vessels of nearly $1 \mathrm{~cm}$ diameter. It was attached to the fundus of the uterus by a thin avascular stalk. Intestines were pushed to the upper half of the abdomen. All other organs including the ovaries were normal. A resection of the mass that was nearly $15 \times 15 \mathrm{~cm}$ with partial omentectomy was done. The histology report confirmed leiomyoma with degenerative changes and a normal omental tissue.

Pradip Dashraath and associates [12] reported a 31-year-old nulliparous woman presenting with progressive abdominal distension and early satiety over the preceding 2 years. On examination, the abdomen was distended with a 40-week sized pelvic abdominal mass. No regional or supraclavicular lymphadenopathy. Cancer antigen 125 level was elevated at 430 units/mL. Computed tomography scan showed 2 large complex solid-cystic masses that appeared to be ovarian in origin. At laparotomy, a giant multilobulated abdominal mass was seen bathed in straw-colored ascites, adherent to the greater omentum via engorged, serpentine vessels. Both ovaries appeared normal. The uterus, which was separate from this mass, was enlarged irregularly by subserosal leiomyomas. infracolicomentectomy was performed, which enabled the mass to be resected en-bloc. On pathologic evaluation, the abdominal mass measured $30 \mathrm{~cm} \times 30 \mathrm{~cm}$ $\times 25 \mathrm{~cm}$ in size. Cut surface demonstrated a whorled appearance with extensive cystic degeneration. Histology was consistent with a benign, parasitic leiomyoma with myxoid and cystic changes. No malignant cells seen in peritoneal fluid cytology.

In comparison to the above four cases that were chosen from the literature, our case is considered as having relatively in common the presentation, the modalities used for diagnosis and the surgical management. However, these cases concern women in the reproductive age which is different from our 55 year old reported case. Furthermore, the above cases consist of a parasitic leiomyoma adherent to the omentum/peritoneum whether our case is a parasitic leiomyoma attached to the mesentery. Note that our case is considered the largest in comparison to all. 


\section{Conclusion}

Parasitic leiomyomas are rare tumors. Imaging modalities help in diagnosis but not always. The definitive treatment is surgical excision, presenting this case due to its rarity and diagnostic difficulties posed.

\section{Conflicts of Interest}

The authors declare no conflicts of interest regarding the publication of this paper.

\section{References}

[1] Robbins, S.L., Cotran, R.S. and Kumar, V. (1984) Pathologic Basis of Disease. 3rd Edtion, WB Saunders, Philadelphia, PA.

[2] Kelly, H.A. and Cullen, T.S. (1909) Myomata of the Uterus. WB Saunders, Philadelphia.

[3] Sreelatha, S., Kumar, A., Nayak, V., Punneshetty, S. and Hanji, N. (2016) A Rare Case of Primary Parasitic Leiomyoma. International Journal of Reproduction, Contraception, Obstetrics and Gynecology, 2, 422-424.

[4] Jones, H.W. and Rock, J.A. (2015) Leiomyomata Uteri and Myomectomy. In: Jones, H.W., Eds. Te Linde's Operative Gynecology, 11th Edition, Wolters Kluwer, Philadelphia, PA, 658-662.

[5] Berek, J.S. (2013) Uterine Fibroids. In: Berek, J.S., Eds., Berek \& Novak's Gynecolo$g y, 15$ th Edition, Lipincott Williams \& Wilkins, Philadelphia, PA, 444-445.

[6] Sarmalkar, M., Nayak, A., Singh, N., Mehendale, M. and Dixit, P. (2016) A Rare Case of Primary Parasitic Leiomyoma Mimicking as Ovarian Mass: A Clinical Dilemma. International Journal of Reproduction, Contraception, Obstetrics and Gynecology, 5, 545-548. https://doi.org/10.18203/2320-1770.ijrcog20160409

[7] Kimberly, A. and Nezhat, C. (2009) Parasitic Myomas. Obstetrics \& Gynecology, 114, 611-615. https://doi.org/10.1097/AOG.0b013e3181b2b09a

[8] Willson, J.R. and Peale, A.R. (1952) Multiple Peritoneal Leiomyomas Associated with a Granulosa Cell Tumor of the Ovary. American Journal of Obstetrics \& Gynecology, 64, 204-208. https://doi.org/10.1016/S0002-9378(16)38757-9

[9] Kale, T., Sanjaykumar, T. and Alnur, Y. () Parasitic Fibroid: A Diagnostic Dilemma.

[10] Iida, M., Ishikawa, H. and Shozu, M. (2016) Spontaneous Parasitic Leiomyoma in a Postpartum Woman. Journal of Obstetrics and Gynaecology Research, 42, 1874-1877. https://doi.org/10.1111/jog.13145

[11] Elagwany, A.S., Rady, H.A. and Abdeldayem, T.M. (2014) A Case of Parasitic Leiomyoma with Serpentine Omental Blood Vessels: An Unusual Variant of Uterine Leiomyoma. Journal of Taibah University Medical Sciences, 9, 338-340. https://doi.org/10.1016/j.jtumed.2014.05.002

[12] Dashraath, P., Lim, L.M. and Huang, Z.W. (2016) Arunachalam Ilancheran, Parasitic Leiomyoma. American Journal of Obstetrics and Gynecology, 215. 